\title{
Thin-shell plastic lenses for space and laboratory applications
}

Herbert W. Schnopper, Russell Ingram, Eric Silver, Marco Barbera, Roberto Candia, et al.

Herbert W. Schnopper, Russell Ingram, Eric Silver, Marco Barbera, Roberto Candia, Finn E. Christensen, Carsten P. Jensen, Suzanne E. Romaine, D. Vernani, Vincenzo Cotroneo, Salvatore Varisco, Maria Antonella Artale, Kristin Kruse Madsen, Alfonso Collura, "Thin-shell plastic lenses for space and laboratory applications," Proc. SPIE 5537, X-Ray Sources and Optics, (3 November 2004); doi: 10.1117/12.559774

EDent: Optical Science and Technology, the SPIE 49th Annual Meeting, 2004, Denver, Colorado, United States 


\title{
Thin shell plastic lenses for space and laboratory applications
}

\author{
Herbert W. Schnopper*a, Russell Ingram ${ }^{a}$, Eric Silver ${ }^{\mathrm{a}}$, Marco Barbera ${ }^{\mathrm{b}}$, Roberto Candia ${ }^{\mathrm{b}}$, Finn E. \\ Christensen $^{c}$, Carsten, P. Jensen ${ }^{c}$, Suzanne Romaine ${ }^{a}$, Dernis Vernani ${ }^{d}$, Vincenzo Cotroneo ${ }^{d}$, \\ Salvatore Varisco ${ }^{\mathrm{b}}$, Maria Antonella Artale ${ }^{\mathrm{b}}$, Kristin Kruse Madsen ${ }^{\mathrm{c}}$, Alfonso Callura ${ }^{\mathrm{b}}$

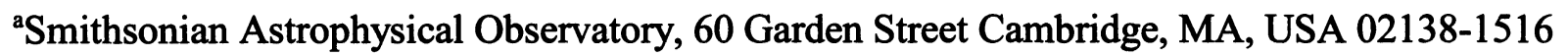 \\ 'Osservatorio Astronomico di Guiseppe S. Viana/INAF, Piazza del Parlamento 1, I-90134 Palermo, \\ Italy \\ 'Danish Space Research Institute, Juliane Maries Vej 30, DK-2100 Copenhagen Ø, Denmark \\ ${ }^{\mathrm{d}}$ INAF-Osservatorio Astronomico Di Brera, via E. Bianchi 46, I-2307 Merate, Italy
}

\begin{abstract}
We have identified an inexpensive, readily available, mechanically stable, extremely smooth, elastic, and mechanically uniform plastic suitable for thin film X-ray optics. Polyethylene terephthalate (PET) is easily deformed without losing its elastic properties or surface smoothness. Most important, PET can be coated with mono- or multilayers that reflect X-rays at grazing incidence. We have used these properties to produce $\mathrm{X}$-ray optics made either as a concentric nest of cylinders or as a spiral. We have produced accurately formed shells in precisely machined vacuum mandrels or used a pin and wheel structure to form a continuously wound spiral. The wide range of medical, industrial and scientific applications for our technology includes: a monochromatic X-ray collimator for medical diagnostics, a relay optic to transport an X-ray beam from the target in a scanning electron microscope to a lithium-drifted silicon and microcalorimeter detectors and a satellite mounted telescope to collect celestial X-rays. A wide variety of mono- and multilayer coatings allow X-rays up to $\sim 100 \mathrm{keV}$ to be reflected. Our paper presents data from a variety of diagnostic measurements on the properties of the PET foil and imaging results form single- and multi-shell lenses.
\end{abstract}

Keywords: X-ray optics, X-ray telescopes, X-ray lenses, X-ray imaging, X-ray collimation, medical diagnostics, microanalysis

\section{INTRODUCTION}

Thin plastic films are manufactured in huge quantities for a wide variety of industrial applications. A process of heating, stretching and rolling is common in most production lines. With time, the rollers become super-polished and the smooth surface is replicated in the contact surface of the plastic film. This super-polished film characteristic is an absolute requirement for producing a highly reflecting $\mathrm{X}$-ray optic. Our current space applications do not require the high angular resolution $(\sim 1$ arcsec half energy width), double reflection, Wolter 1, imaging that characterizes the $\mathrm{X}$-ray telescope on CHANDRA X-ray Observatory. Instead, our requirements are satisfied by a conical approximation to Wolter 1 optics and, in some cases, a single conical reflection meets our needs.

Cost and mass requirements have led us to seek ways of exploiting thin plastic film technology for future hard X-ray lenses and telescopes. We start with a material that is already smooth on a scale that is appropriate for mono- or multilayer deposition. The plastic has a strong affinity for its coating which can be scraped with a sharp edge, but cannot be "brushed" away. Perhaps the most important characteristic of plastic film is that it can be coated before it is formed into a lens.

We have constructed and tested several prototype X-ray lenses and have already reported some of our results (Schnopper, et al $\left.^{1,2,3}\right)$. Single element, cylindrical shells and cylindrical spiral lenses have a point-to-point imaging, single reflection geometry that is an approximation to a point-to-point imaging elliptical lens. Lenses formed as cones approximate a parabola.

*hschnopper@head.cfa.harvard.edu; phone 1617 486-7763: fax 1617496 7577; http://cfa-www.harvard.edu 
State-of-the-art coating facilities are well established at SAO and at DSRI. Mono- or multilayer coatings of C, Si, Ni, $\mathrm{W}$ and $\mathrm{Pt}$ can be deposited on test films. At SAO the films are wrapped around the inner wall of the $65 \mathrm{~cm}$ diameter sputtering chamber. At this distance from the cathode, we can deposit a uniform layer at a surface temperature that avoids surface damage. Surface stress is avoided by coating the foil material on the "back" side before coating the reflecting side. This also "locks' both surfaces against moisture infusion. Tests repeated over a period of $\sim 1$ year on coated and shaped foil samples demonstrate the robustness of the coating. Test optics have been temperature and vacuum cycled in the course of transport to and from testing in various beam line facilities. The imaging results obtained from them are reproducible.

\section{SURFACE PROPERTIES OF COATED PET FILM}

\subsection{Atomic force microscopy (AFM)}

AFM scanning reveals surface structure on scales from $<1 \mu \mathrm{m}$ to $50 \mu \mathrm{m}$ (see Figure 1). These tests were made at the Osservatorio Astronomico di Brera. The sample is $175 \mu \mathrm{m}$ PET coated with a $100 \mathrm{~A}$ of W. The sample is a portion of the shell that was mounted in MHM-1, one of two cylindrical mandrels that is used to form a $141 \mathrm{~mm}$ diameter cylindrical lens. The $1 \mu \mathrm{m}$ scan shows typical features with an amplitude of $2-3 \mathrm{~nm}$ separated by $0.2 \mu \mathrm{m}$. Similar results are obtained from the $10 \mu \mathrm{m}$ scan. The fine structure on the $50 \mu \mathrm{m}$ scan is similar. There are, however, a scattering of $10 \mathrm{~nm}$ high features that are up to several $\mu \mathrm{m}$ wide and less than $1 \mu \mathrm{m}$ wide. The sample came from a cylindrical shell that was tested at the $\mathrm{x}$-ray beam pipe in Palermo which was transported back and forth between Cambridge and Palermo several times before being sent to Brera. The sample was exposed to room conditions several times and could easily have become contaminated by dust particles. The measured surface roughnesses $\left(\sigma_{\mathrm{rms}}\right)$ obtained for the 1,10 and $50 \mu \mathrm{m}$ scans are $0.65,0.85$ and $0.87 \mathrm{~nm}$, respectively. They are obtained from combined Power Spectral Density (PSD) results and are presented in Figure 2.

\subsection{WYCO optical interferometry}

These data were also obtained at Osservatorio Astronomico di Brera. WYCO scans cover about $6 \mathrm{~mm}$ along the surface and are sensitive to sub- $\mu \mathrm{m}$ features. Two scans are reported. The surface roughnesses for the "good" and "bad" scan $s$ are 0.72 and $1.43 \mathrm{~nm}$, respectively. The PSD results are shown in Figures 2 and 3.

\subsection{Small angle $X$-ray scattering}

We tested a W coated plastic at the small angle X-ray scattering facility at the Danish Space Research Institute (DSRI) (Figure 4). A Cu K $\alpha$ beam produced by a micro-focus, rotating-anode X-ray tube is collimated by a slit system and made highly monochromatic and parallel by a 5-bounce, channel-cut Si crystal. We adjusted the incident angle of the beam on the sample to be $0.6 \mathrm{deg}$ to make a narrow footprint along the sample that is just short of the $100 \mathrm{~mm}$ length of the shell. This avoids spurious scattering from the edges of the sample. Our first measurements were made with the shell under vacuum in a mandrel. The results were acceptable, but the vacuum caused the plastic to deflect slightly into the $0.78 \mathrm{~mm}$ pumping holes and this introduced an additional scattering component. Since our goal was to establish the scattering properties intrinsic to the plastic, we made additional tests on a sample held flat to $+/-1 \mu \mathrm{m}$ in a porous ceramic vacuum chuck. The half energy width (HEW) improved from 122 to 34 arcsec. Both results are shown in Figure 5.

\section{MECHANICAL ASPECTS}

\subsection{Shells with cylindrical or conical symmetry made using mandrels}

Depending upon the application, we form the shells in either cylindrical or conical mandrels. They are then transferred to a structure and nested into a lens. A typical mandrel mounted with a plastic shell is shown in Figure 6. It has an inner diameter of $141 \mathrm{~mm}$. The mandrel body is drilled with a number of fine radial holes. An outer cylinder fitted with a pumping port slides over the mandrel body to make a vacuum chamber. Before being mounted in the mandrel, we cut the plastic film to the correct width and a length that will leave a small gap between the ends when the shell is formed The plastic film has a certain restoring force when it is formed in the mandrel. If unrelieved, the residual stress will lead to a less than perfect figure after the ends are joined and the shell is removed from the mandrel. We avoid this distortion by annealing the foil in a temperature-controlled oven before the joining the ends. We increased the temperature slowly to a value just below the glass transition temperature, $T_{g}$, and then maintain the temperature for a period of hours. To complete the shell, we draw the foil to the shape of the inner surface of the mandrel by vacuum pumping. Next, we connect the ends with a thin, double sticky, Kapton tape placed between a narrow strip of thin plastic material that overlaps ends of the foil. 
We have used two methods to nest the shells. Our first concept was to cage each shell in a precise cylindrical array of pins that span two spoked wheels. Our first small spiral lenses were made this way and our very first images were made with the 20 shell lens shown in Figure 7. Our second design eliminated much of the structure and substituted a single, 32 spoked, wheel with a groove cut to accommodate one edge of the shell in each of the spokes. The groove is made slightly wider and deeper than the portion of the foil that penetrates the wheel to allow for buffers of epoxy glue on either side and at the end of the shell (Figure 8).

\subsection{Spiral shells}

We returned to our wheel and spoke technology to make spiral lenses. That design not the best approach for cylindrical lenses, but it offered us a significant cost advantage in forming spiral lenses since they do not require mandrels. Two examples of small spiral lenses are shown in Figure 9 and a prototype large spiral in shown in Figure 10.

The prototype structure has been produced with tight tolerances $( \pm 1 \mu \mathrm{m})$ and will be fitted with seven turns of a W coated plastic spiral. We will evaluate the lens from the quality of the X-ray imaging results scheduled to take place at the XACT facility in Palermo in October 1994.

\subsection{Shell figure metrology}

Our mandrels are made in-house on a CNC lathe with a nominal precision of $\pm 4 \mu \mathrm{m}$. Each pumping hole leaves a burr on the otherwise smooth surface and after machining, the surface was honed to remove the burr. We measure the shape of the inner surface with a profilometer located at the Osservatorio Astronomico Di Brera. The best results were obtained at the center of the mandrel and are shown in Figure 11. We have concluded that a switch to diamond turning technology was necessary to produce mandrels with appropriately small $( \pm 0.1 \mu \mathrm{m})$ long rang slope errors.

\section{IMAGING RESULTS}

\subsection{X-ray Astronomy Calibration and Testing Facility (XACT) at the Palermo Observatory}

We have chosen this facility (Figure 12) to evaluate the imaging properties of our plastic lenses. XACT features a $\sim 17.5 \mathrm{~m}$ vacuum beamline, an interchangeable anode $X$-ray source and a $40 \mathrm{~mm}$ diameter channel plate detector. Our X-ray tube has been modified to allow a maximum of $20 \mathrm{kV}$ to be applied to the anode. The side ports in the beamline allow us to insert lenses and an alt-az mount (shown in Figure 8) at intervals of $1 \mathrm{~m}$ along the beam line. Our Manson X-ray source allows access to up to four different anodes and filters without breaking vacuum. The $8 \mathrm{~mm}$ face of the anode is illuminated by a hairpin W cathode and makes a $45 \mathrm{deg}$ angle to the beamline direction. Although there is no monochromator in the facility, we can control the energy content of the emerging beam through the choice of filters and anode voltage. A rough knowledge of the spectral content of the X-ray beam can be obtained by mounting a thin window, gas filled, proportional counter on a second port in the X-ray tube. That port makes an angle of $90 \mathrm{deg}$ to the beamline and also views the anode at a 45 deg angle.

\subsection{Imaging the source through a pinhole}

We know that the Manson Source anode does not provide a precise point source of X-ray illumination. If the extent of the source distribution is known, then we can remove that contribution to the X-ray image formed by the lens. We have found that the extent of the source distribution depends upon the choice of anode and high voltage. By inserting a $200 \mu \mathrm{m}$ pin hole $\sim 850 \mathrm{~mm}$ from the $\mathrm{C}$ source we form a $\sim 20: 1$ magnification of the source distribution on the detector (Figure 13). The 40 $\mathrm{mm}$ diameter detector sees only the central $2 \mathrm{~mm}$ of the $8 \mathrm{~mm}$ diameter source. In the future, we will scan the detector and stitch together a complete image of the source distribution.

\subsection{Images obtained from a $141 \mathrm{~mm}$ diameter cylindrical lens held in a mandrel}

We obtained images made at various X-ray energies with the lens shown in Figure 6. The lens is mounted halfway between the source and the detector. After an alignment in pitch and yaw, we can adjust the position of the detector along the beamline to find the best image. We also obtain the detector background distribution with the X-ray source turned off. Our analysis of the background subtracted data produces an image histogram, a projected image, a radial profile curve and an encircled energy curve derived from the profile. We derive the full width at half maximum (FWHM) from the radial profile and the half energy width (HEW) from the encircled energy (Figure 14). In some cases, we have modeled the contributions to the image that come from the non-point source distribution and from the cylindrical lens approximation to a true imaging elliptical lens. In Figure 14, we show the data obtained in June, 2002 using a $\mathrm{C}$ anode ( $\mathrm{C} \mathrm{K} \alpha=0.25 \mathrm{keV}$ ). Two model curves are 

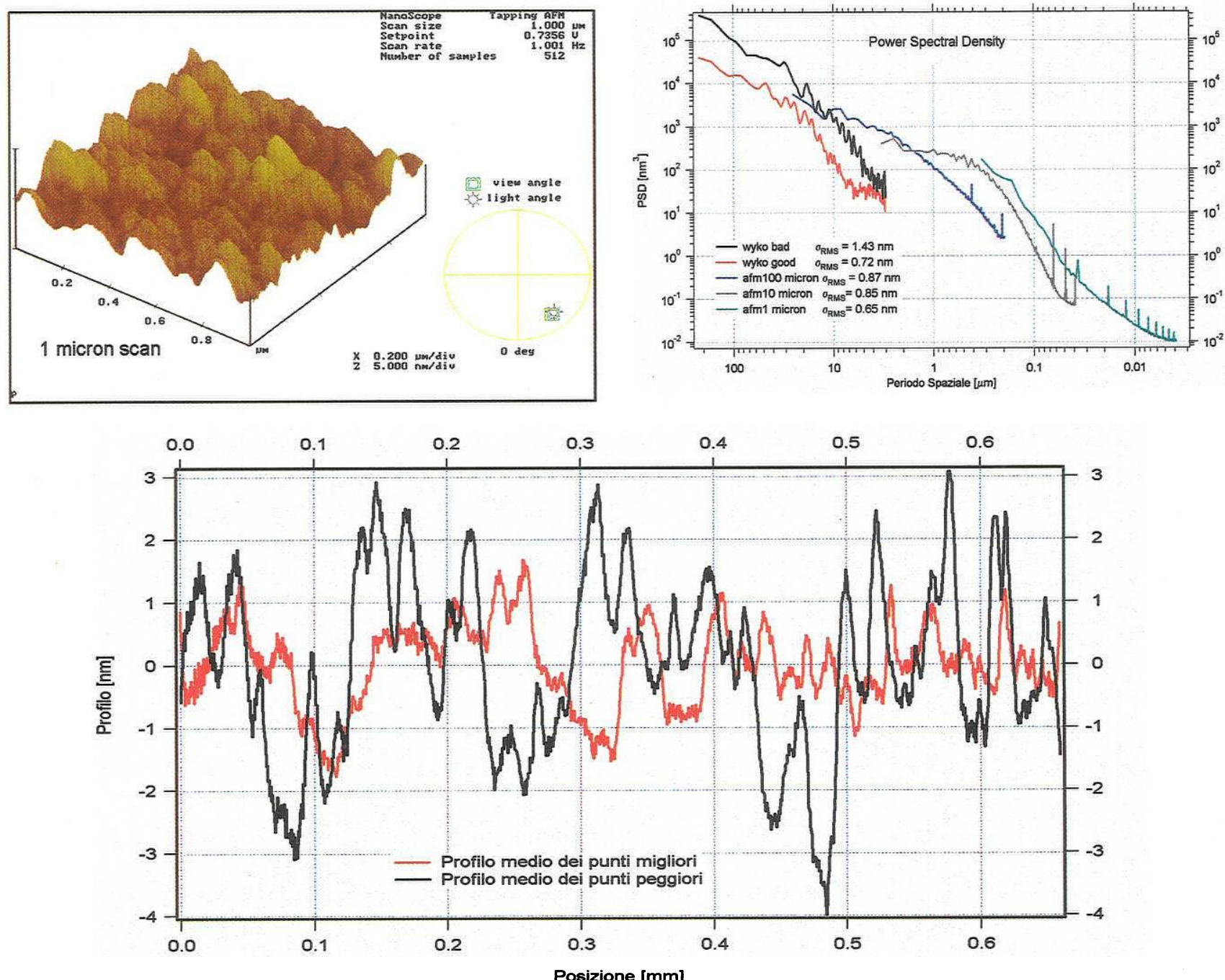

Figure1 (top left). Data from a $1 \mu \mathrm{m}$ AFM scan from a portion of a $175 \mu \mathrm{m}$ PET shell coated with $175 \AA$ of W. Images from the shell were obtained at the $\mathrm{X}$-ray beam line in Palermo. Data from 10 and 50 and $100 \mu \mathrm{m}$ scans show similar small scale features. The $50 \mu \mathrm{m}$ scan also shows widely scattered large features that are assumed to be dust.

Figure 2 (center). WYCO interferometer scans from "good" and "bad" regions on the same sample.

Figure 3 (top right). Power Spectral Density (PSD) curves obtained from the AFM and WYCO data. The 1 and 10 $\mu \mathrm{m}$ AFM data and the "good" WYCO data yield surface roughnesses in the $0.7-0.9 \mathrm{~nm}(7-9 \AA)$ range. These values are somewhat larger than we would like to form the best images and some of the additional roughness may be attributed to wear and tear during transport.

Figure 4 (bottom). A view of the small angle scatterometer located at DSRI. The main elements in the beam path are indicated. The sample, a coated cylindrical PET shell, is under test. 

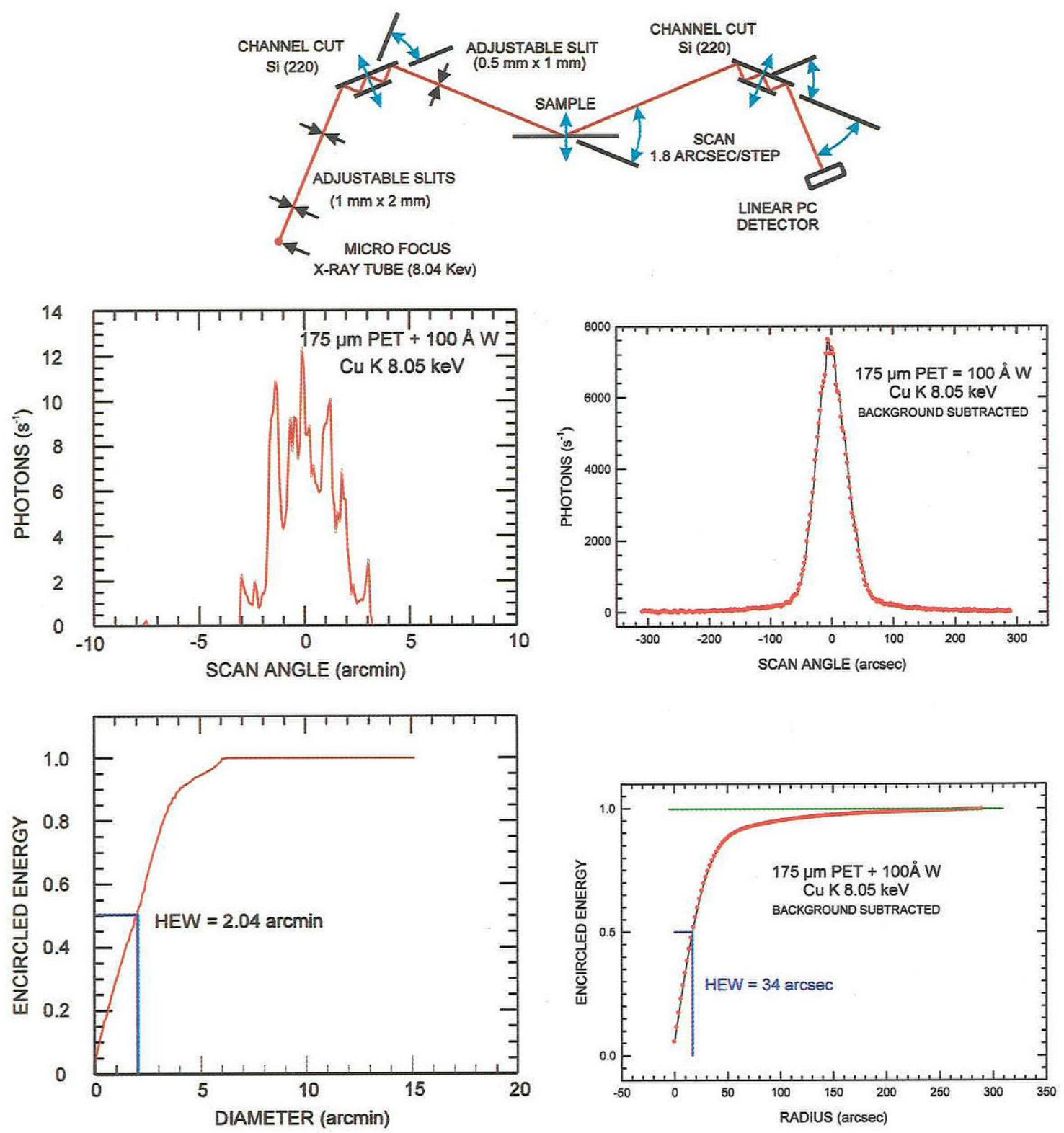

Figure 5a (top). The components of the DSRI Scatterometer shown in Figure 4. The arrows indicate the various linear and angular scans that are used to align the instrument and then to collect data. The instrument is aligned in the $(1,-1)$ configuration (not shown) and then set to take data in the $(1,+1)$ position as shown. The channel cut crystals provide a parallel monochromatic beam at $8.04 \mathrm{keV}(\mathrm{Cu} \mathrm{K \alpha})$. With the sample in place, the arm carrying the second crystal and the detector scans the beam reflected from the sample. The ideal result should be a peak equivalent to the $(1,-1)$ curve since the reflection from the sample "flips" the beam back into a non-dispersive configuration. The pencil beam defined by the slits can be adjusted to a "footprint" that is any length equal to or shorter than the length of the shell. Deviations from the $(1,-1)$ curve are introduced by small angle scattering and figure errors.

Figure $5 \mathrm{~b}$ (left middle and left bottom). The small angle scattering curve from the shell in the mandrel shown in Figure 4 . The individual peaks are from smooth, long wavelength structures along the footprint. The sharp features indicate the good smoothness of the surface, while the distribution of the peaks can be caused by inaccuracies in the mandrel figure or foreign matter between the shell and the mandrel. We suspect the latter.

Figure 5c (right middle and left bottom). When the mandrel is replaced by a precision vacuum chuck, the scattering curve shows only a single peak. Half of the scattered energy is contained within a 34 arcsec diameter. 


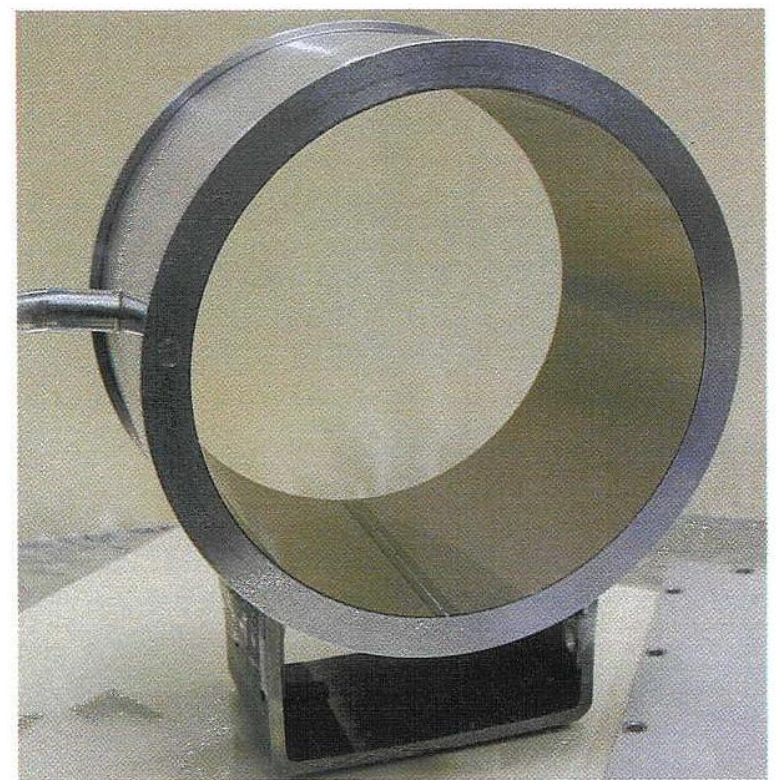

Figure 6. A $156 \mathrm{~mm}$ diameter vacuum mandrel mounted with a W coated PET shell. The joint is at the bottom.

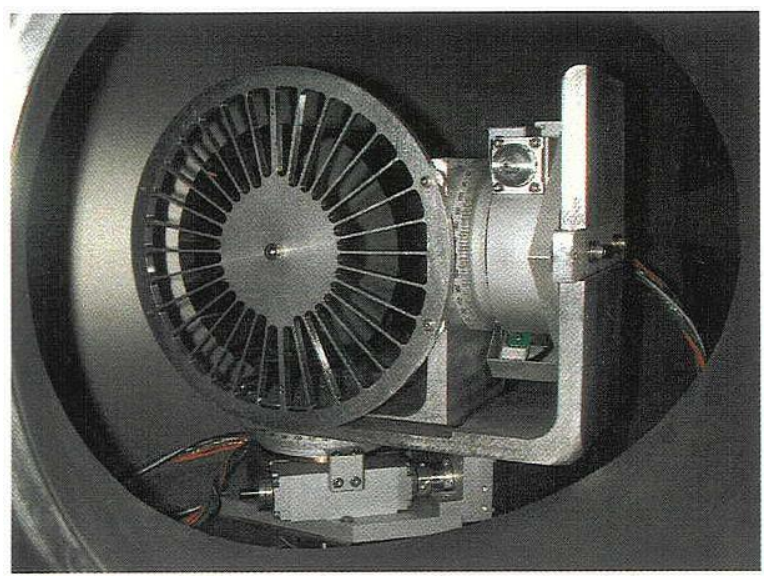

Figure 8. A $141 \mathrm{~mm}$ diameter lens mounted in a grooved wheel. The wheel is attached to an Alt-Az mount ,turned to an angle of $45 \mathrm{deg}$, inside a $60 \mathrm{~cm}$ diameter section of the Xray beam pipe at the $17 \mathrm{~m}$ long XACT facility in Palermo.

Figure 10 (right). This large spiral prototype lens is wound with seven turns of $\mathrm{W}$ coated PET plastic. The winding are between two rings. Twenty-four ribs in the front and back wheels support a $50 \mathrm{~mm}$ wide, single strip of plastic. The pins are $1.27 \mathrm{~mm}$ in diameter and the spacing between the shells is $2 \mathrm{~mm}$. A 6 in scale is placed in front of the lens.

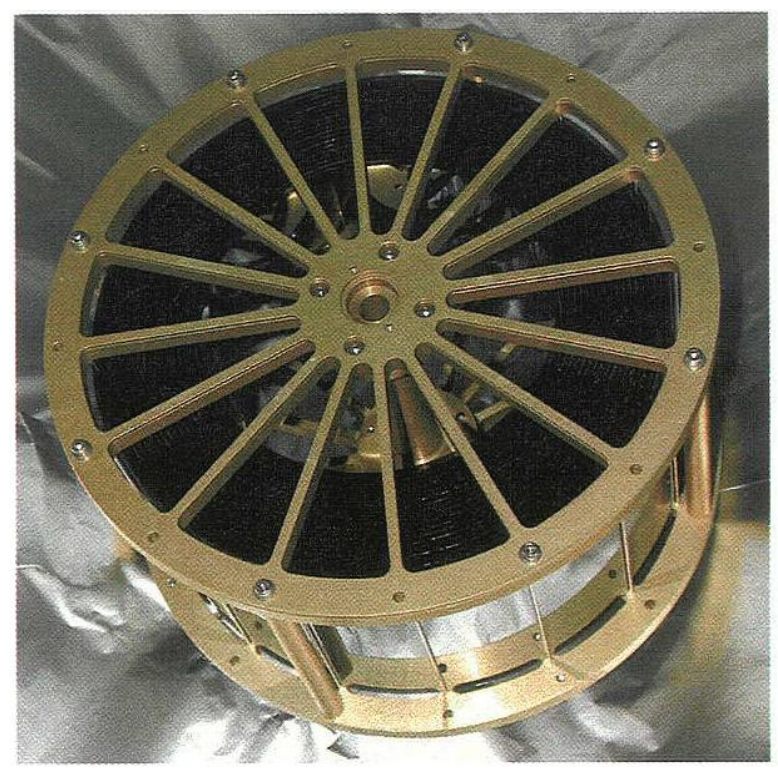

Figure 7. A $175 \mathrm{~mm}$ diameter lens mounted with $20 \mathrm{~W}$ coated PET plastic shells.

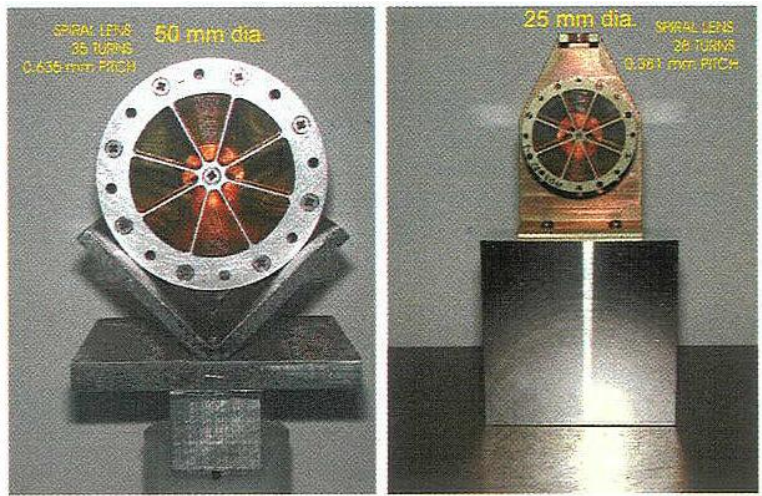

Figure 9. Two spiral lenses that have been tested at the XACT facility in Palermo. Their plastic ribbons are coated with an evaporated layer of $\mathrm{Au}$. Better results will be obtained when they are replaced with $\mathrm{W}$ sputtered ribbons.

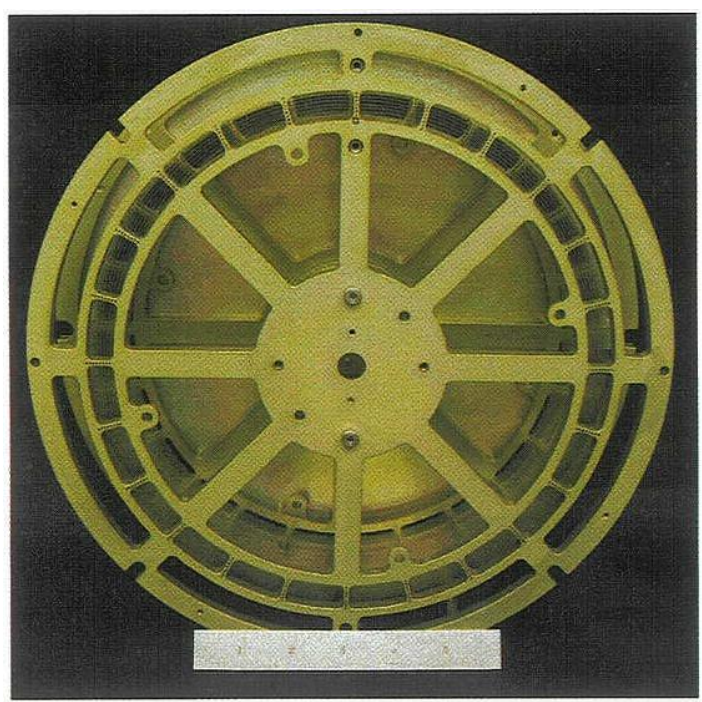




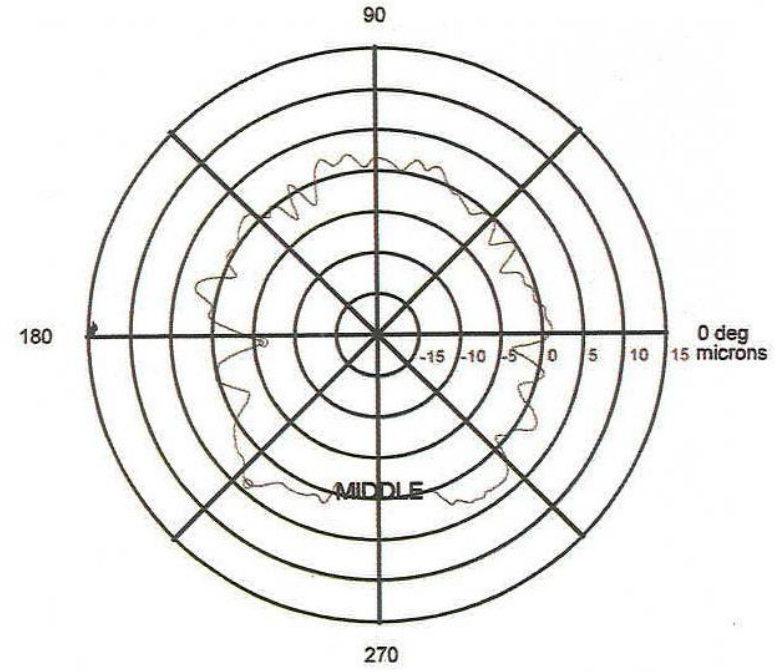

Figure 11. CMM metrology results for one of our cylindrical mandrels. The groove is at the bottom.

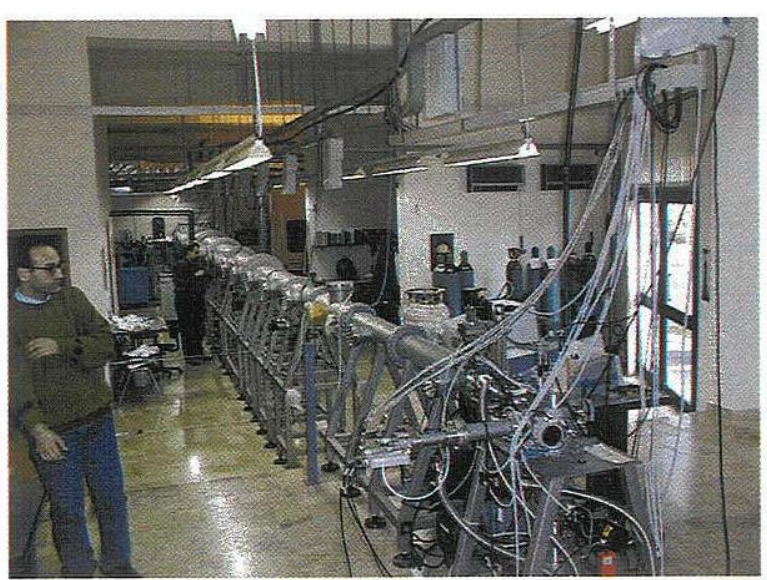

Figure 12. The XACT Facility in Palermo. The X-ray tube is at the near end.

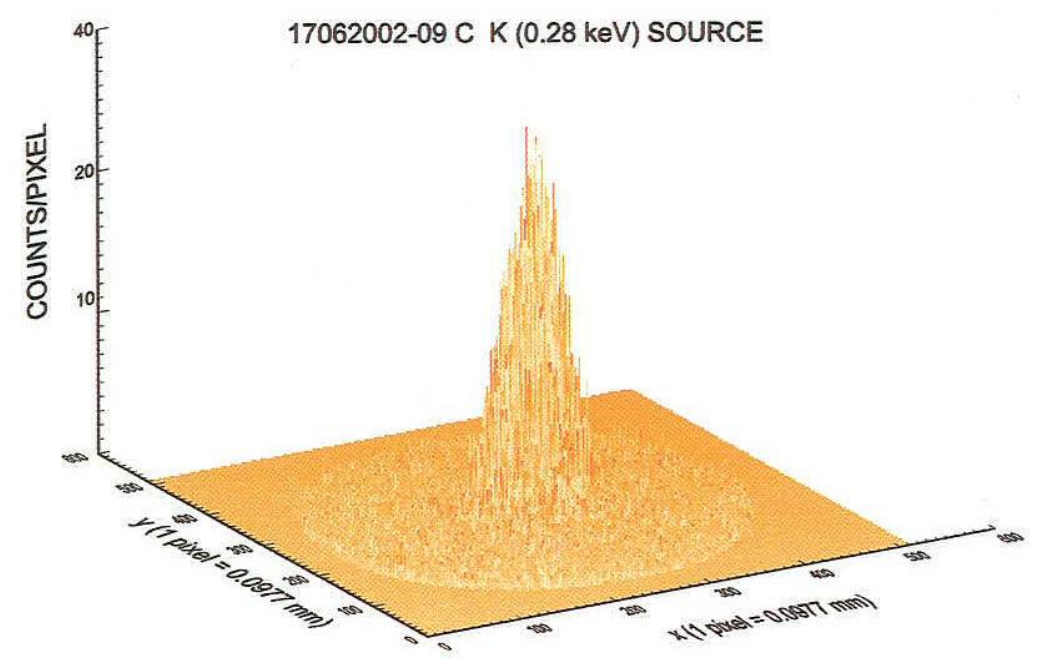

Figure 13. The central portion of the histogram clearly defines the primary source C K X-ray emission from electrons focused on the target.
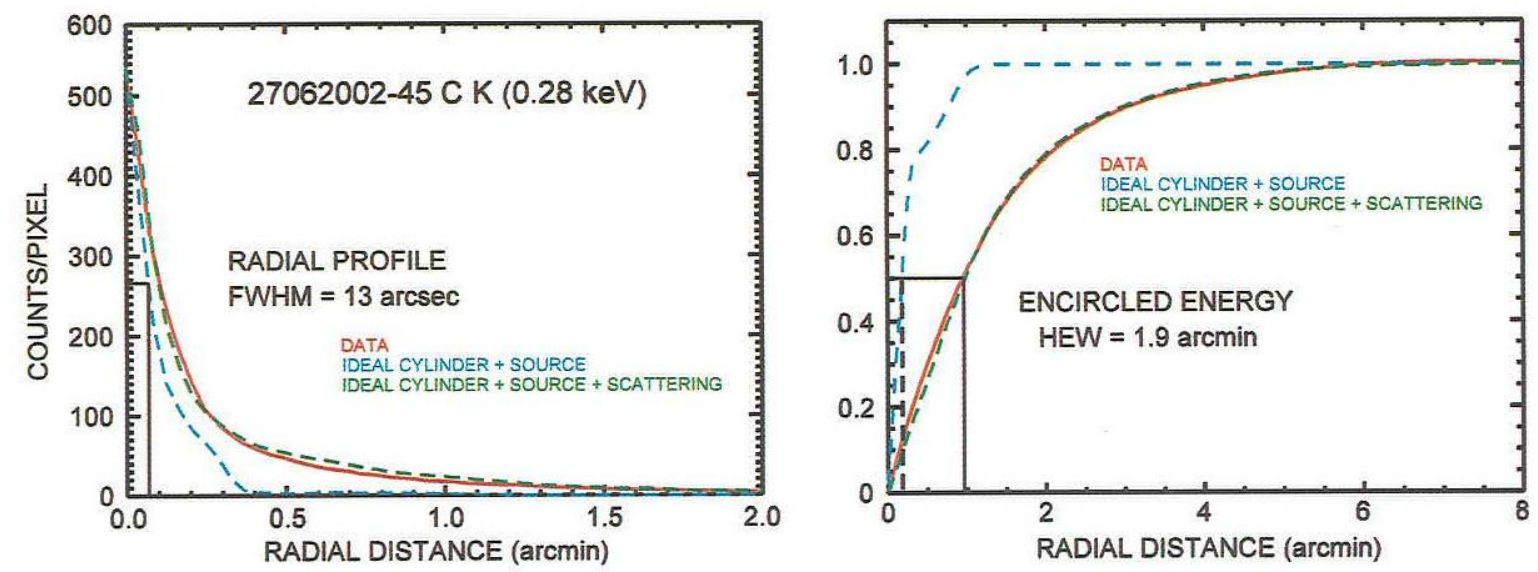

Figure 14. Figure 14 (above). Data from a $\mathrm{C}$ target that has been modeled to include effects from the cylindrical geometry and small angle scattering from surface imperfections. 
shown in each: both include a component that accounts for the cylindrical geometry and the source extent and the second includes small angle scattering model. As expected, the small angle scattering has only a minor effect on the radial profile FWHM, but has a major one on the encircled energy HEW. Next, we show more recent data taken in May 2004 with a Cr anode (Figure 15). Here, we obtain a FWHM $=7$ arcsec which is a very good match with the contributions from a point source and the cylindrical geometry. Our HEW $=3.4$ arcmin is much greater than would have been expected based on previous results. We know that the detector was behaving erratically during that period. Only a small increase in background leads to a large increase in the HEW while the FWHM is only marginally changed.

\subsection{Images obtained from the 25 and $50 \mathrm{~mm}$ diameter cylindrical spiral lenses}

Testing the small cylindrical spiral lens shown in Figure 9 presented us with several problems. The lens was originally design to closecouple ( $1 \mathrm{~m}$ ) an SEM with our microcalorimeter. The spiral shell spacing and length was dictated by that geometry. Four the source-tolens distance of $\sim 8 \mathrm{~m}$ at the XACT facility, the separation between the spiral turns is too large to block straight-through rays. Some of the straight-through rays would hit the detector unless we blocked the inner portion of the lens and created a shadow on the detector that was large enough to form an image from the reflected rays. The occulting disc seen in Figure 9 blocks the inner third of the shells. There was also a problem of intensity. The net collecting area of the spiral lens is much smaller than what we obtained from the, single shell, cylindrical lens. Despite these difficulties, we obtained a FWHM of $7 \operatorname{arcsec}$ with a $\mathrm{Cr}$ anode $(\mathrm{Cr} \mathrm{K} \alpha=5.4 \mathrm{keV})(\mathrm{see}$ Figure 16). We could not obtain a meaningful HEW from the sparse data.

Our $50 \mathrm{~cm}$ spiral lens is able to collect a significantly greater number of X-rays. Potential straight through X-rays are blocked by the central hub. The sharp image that we obtained is shown in Figure17. It shows the predicted dark region at the center of the image clearly and we interpret this as evidence that the shells are very well aligned to the axis of the lens. The image of an elliptical spiral would be a circle about the "ideal" focal point. In our case, each point on the cylindrical spiral is tangent to an ellipse with foci at the focal spot and the focal point for its own ellipse. The diameter of the unfilled circle is the same for each ray originating on-axis, but there will be a distribution of detected events that falls off to larger diameters. Off-axis X-rays originating in the slightly extended X-ray source distribution and surface roughness both cause the region inside the circle to fill in. We note that none of the reflected rays should cross the axis.

\section{APPLICATIONS}

\subsection{Relay lens}

We built the $50 \mathrm{~mm}$ spiral lens as a means to couple the Dewar containing our X-ray microcalorimeter with an SEM and it was tested with our $\mathrm{Si}(\mathrm{Li})$ detector ${ }^{4}$. The plastic spiral is coated with $\mathrm{Au}$ and we knew that close coupling would cause the grazing angles on outer shells to exceed, $\theta_{c}$, the critical angle for total reflection. The radius at which $\theta_{c}$ is exceeded gets smaller with increasing X-ray energy. We can solve the $\theta_{c}$ problem by using multilayered lenses. We obtained two sets of data during the $\mathrm{Si}(\mathrm{Li})$ test (Figure 18). One is from a series of characteristic X-ray lines and the other from a continuum. Absorption in the protective, $50 \mu \mathrm{m} \mathrm{Be}$, window in the $\mathrm{Si}(\mathrm{Li})$ Dewar causes the turnover in counting rate at energies below $\sim 1.5 \mathrm{keV}$. X-rays with energies above $\sim 9 \mathrm{keV}$ exceed the critical angle for most of shells. We have much thinner windows that separate the temperature stages in the microcalorimeter Dewar. They allow sensitivity down to a few hundred eV. We have used the $50 \mathrm{~mm}$ spiral lens successfully to couple our microcalorimeter with the X-ray emitting region in the NIST Electron Beam Ion Trap (EBIT) $)^{4,5}$

Relay lenses can have conical as well as cylindrical symmetry. This is particularly important when the source of the X-rays releases, in addition to a huge X-ray flux, an enormous energy in the form of heat and particles that will destroy nearby instruments. For example, we have suggested that an asymmetrically configured, plastic, conical lens, placed downstream from the interaction volume in the National Ignition Facility (NIF) at LLNL, would survive long enough to capture meaningful data. Our solution is relatively inexpensive when compared with the cost of replacing highly polished glass lenses after each shot.

\subsection{Medical}

Two of us (HWS and SR) have already reported on an X-ray lens that will produce a quasi-monochromatic beams of X-rays suitable for dual energy subtraction angiography studies ${ }^{6}$. Our original design is based upon an array of multilayered Si substrates. Multilayers with a roughness $\sim 2 \AA$ exist ${ }^{7}$ and $2 \mathrm{~d}$-spacings of $25 \AA^{7}$ and $20 \AA^{8}$ are under development (smaller by a factor of 2 than the value we incorporated in our original deign ${ }^{6}$ ). When we add that capability to our ability to make plastic spiral lenses, our original concept for angiography becomes more of a practical reality. We use our multilayered spiral technology to design lenses (Figure 19) that will produce quasi-monochromatic beams of Mo K (17.5 keV) and Ag K (22.2 $\mathrm{keV}) \mathrm{X}$-rays for mammography and beams of $\mathrm{Ba} \mathrm{K} \alpha_{1}(32.2)$ and $\mathrm{La} \mathrm{K} \alpha_{1}(34.4 \mathrm{keV})$ X-rays for angiography. 

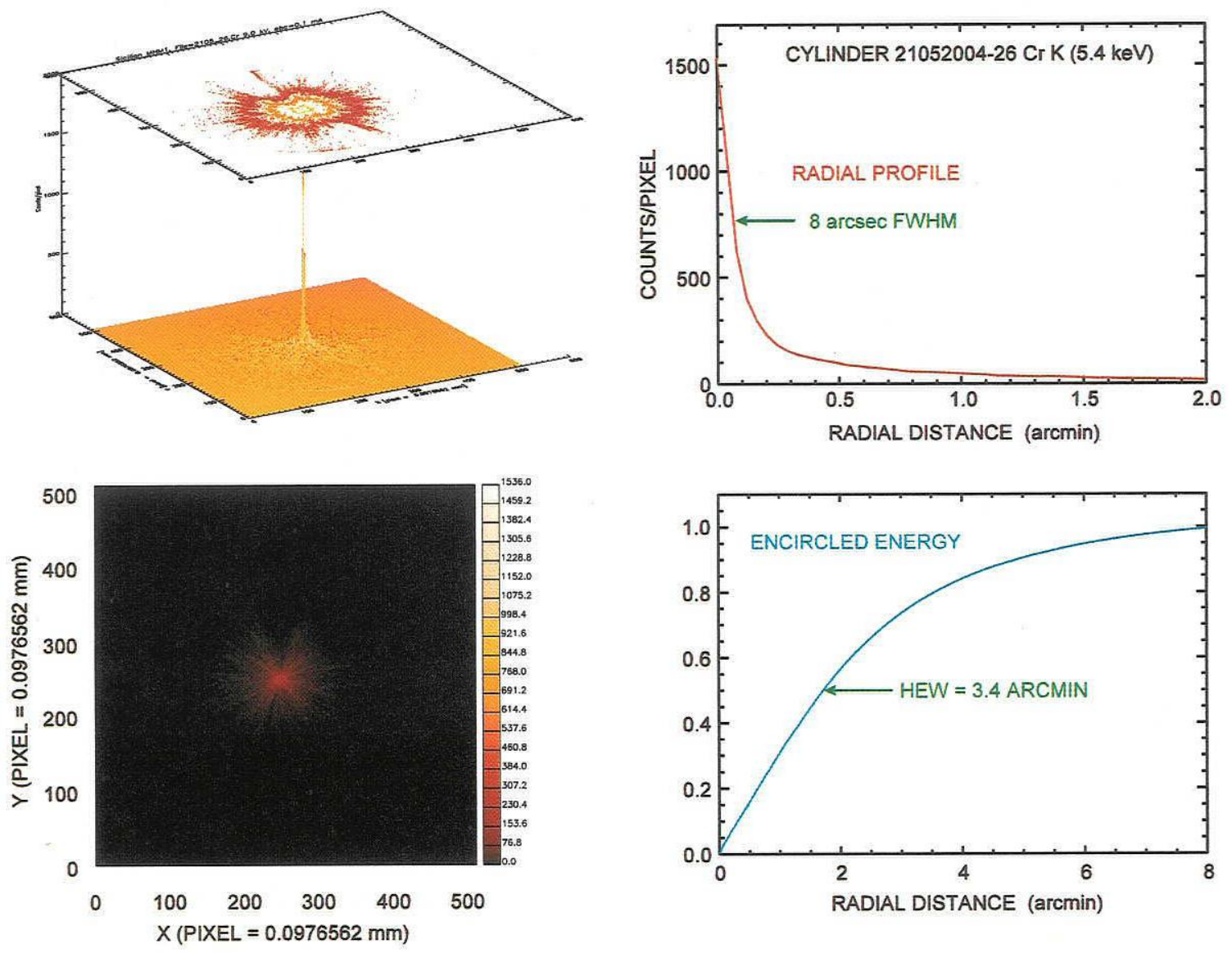

Figure 15 (left). The full analysis of reflection data from $141 \mathrm{~mm}$ diameter cylindrical lens illuminated by a $\mathrm{Cr}$ anode. A noisy detector caused the unexpectedly large HEW. The FWHM is less affected by the noise. The central spike in the image radial profile is dominated by the geometry of the cylindrical lens.
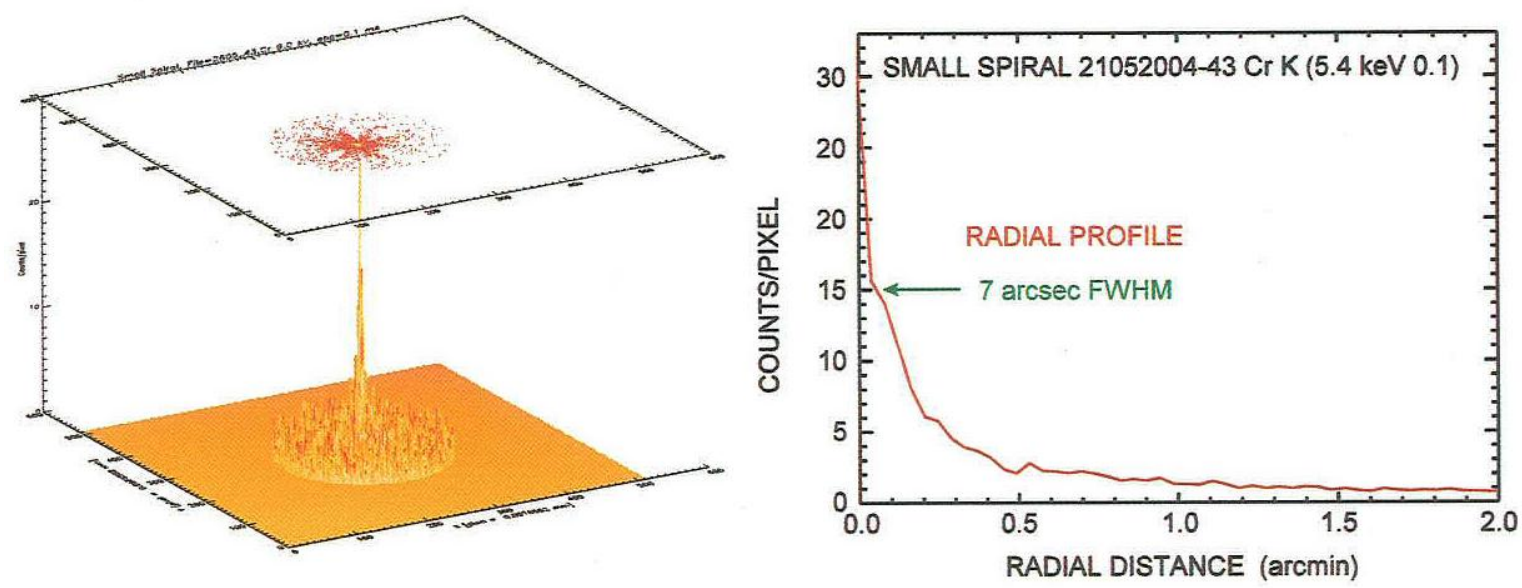

Figure 16. An analysis of an image formed by the $25 \mathrm{~mm}$ diameter spiral lens illuminated by a Cr K X-rays. The image has been cut-off at a point where straight through X-rays hit the detector. 


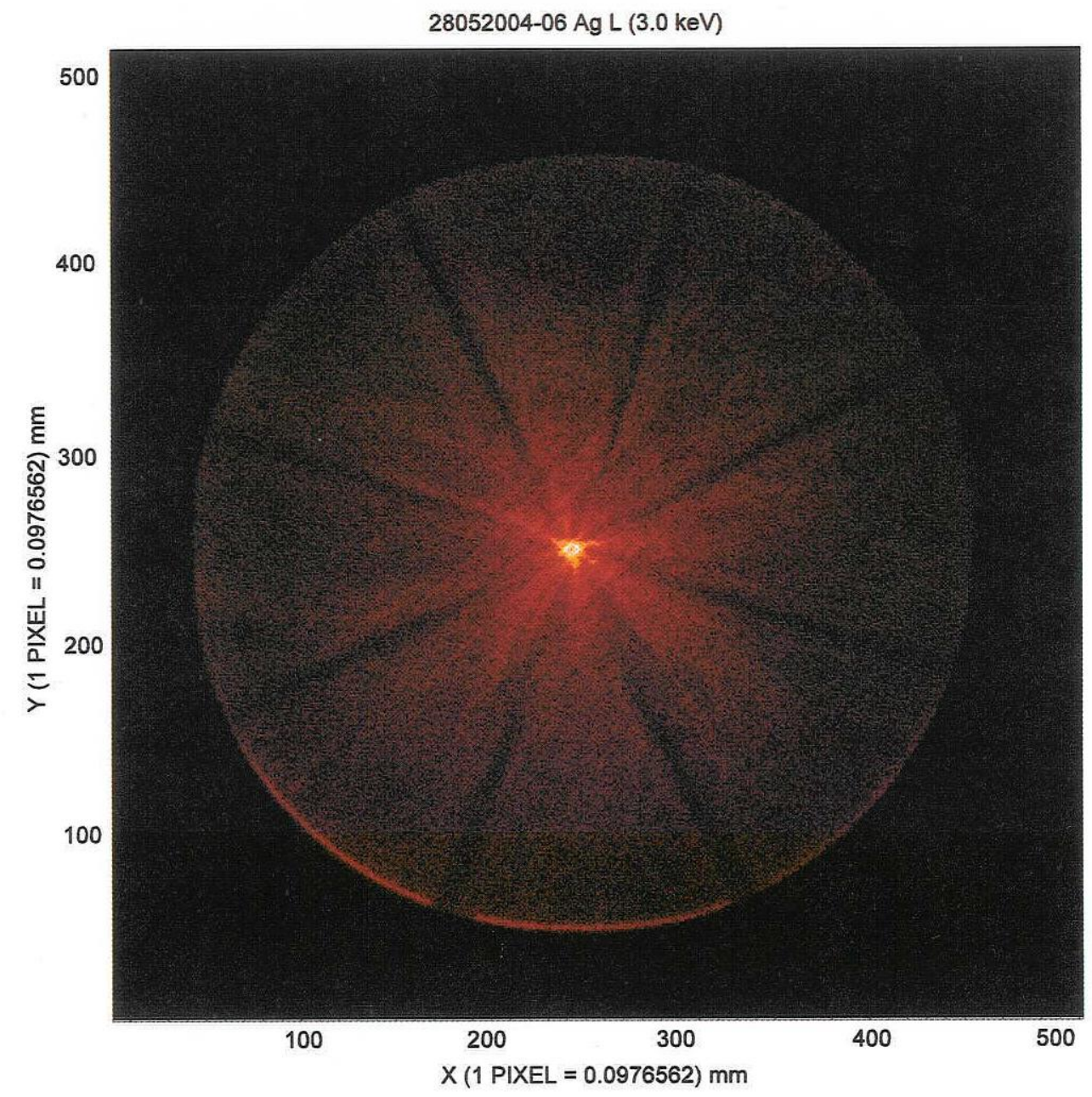

Figure17. A projection of an image made by the $50 \mathrm{~mm}$ spiral lens. Just visible in the center of the image is a small dark spot that is a characteristic of spiral lenses. Since the lens is cylindrical instead of elliptical, the hole is partially filled.
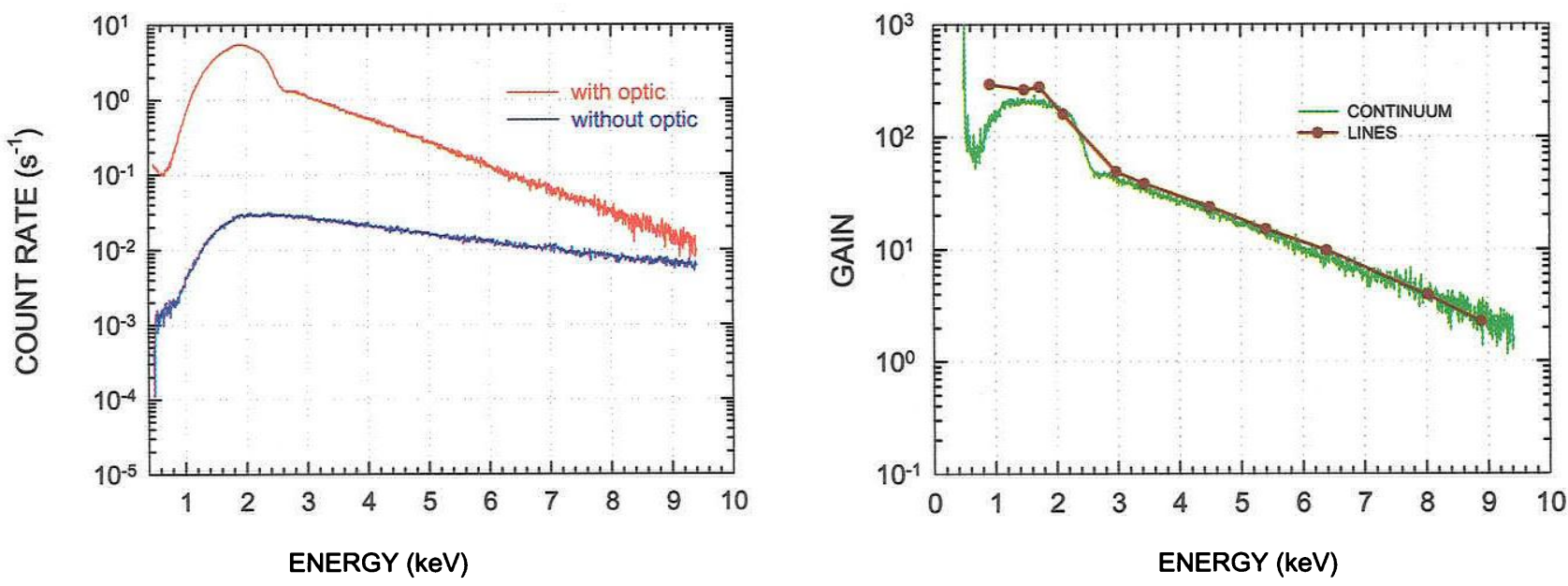

Figure 18. We obtained this data from a $\mathrm{Si}(\mathrm{Li})$ that was coupled to our scanning electron microscope with the spiral lens halfway between them. The maximum gain came at $2 \mathrm{keV}$. Above that energy the incident angle begins to exceed the critical angle. 


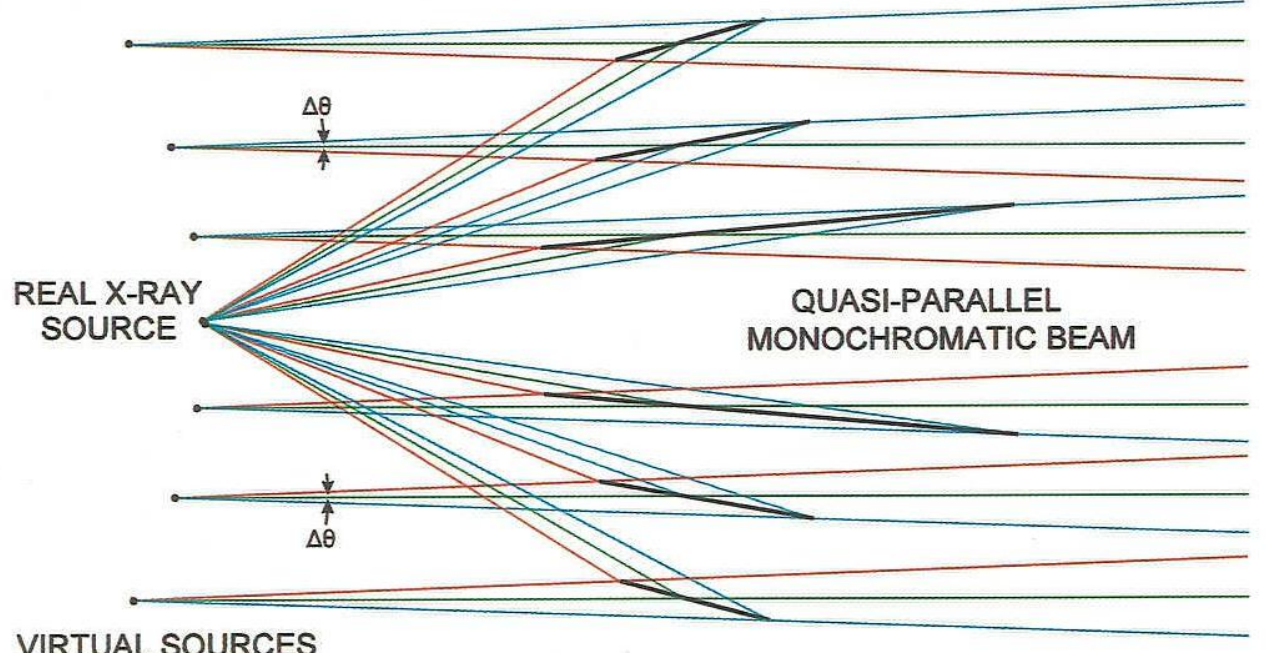

VIRTUAL SOURCES

\section{CONICAL SPIRAL X-RAY MONOCHROMATOR}

Figure 19. Spiral lenses can be coated with multilayers to produce quasi-parallel beams of monochromatic $\mathrm{X}$-rays. X-ray optics coated with the smallest multilayer spacings now available (2d $=20 \AA$ ) can be used in a variety of space, medical and industrial applications. We show, in a highly exaggerated schematic, how a continuous band of multilayered plastic can be formed into a monochromator. In practical applications it is more convenient to use a constant width plastic ribbon.

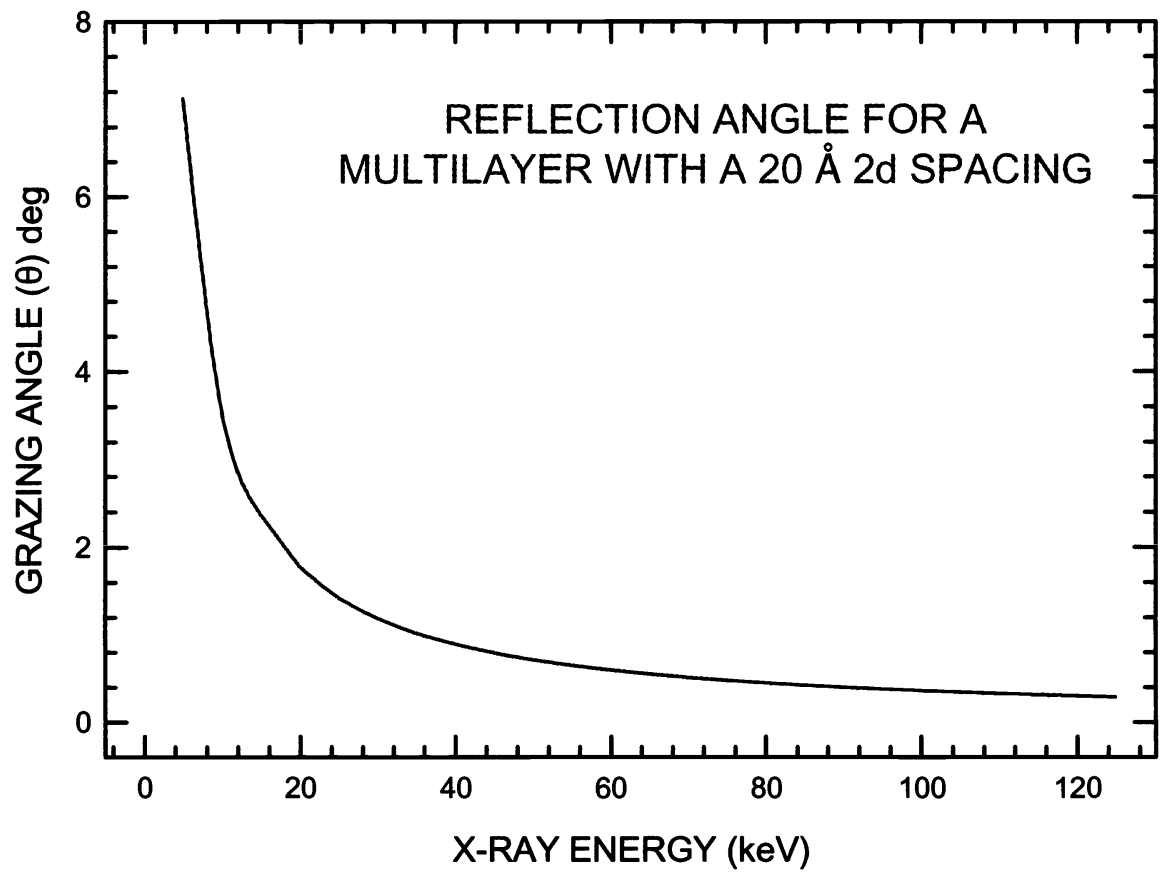

Figure 20. Small d-spacing multilayers are the essential ingredient in the development of high energy $\mathrm{X}$-ray optics. For any choice of substrate or lens geometry, the high energy X-ray reflectivity is determined by the multilayer design and the smoothness of the substrate. To put this figure in perspective, the inner shell on the $10 \mathrm{~m}$ focal length $C H A N D R A$ telescope had a minimum grazing angle of $0.45 \mathrm{deg}$ and a maximum energy response of $10 \mathrm{keV}$. 
We can also design a focusing geometry that will, for example, focus a high energy X-ray beam into a small spot. Although grazing angles will be quite small, $\sim 0.2 \mathrm{deg}$ for a $0.1 \AA(\sim 120 \mathrm{keV})$ beam (Figure 20$)$, the benefits of using a focused beam for the patient are large.

\subsection{High energy astrophysics}

All of the authors have a major interest in high energy astrophysics. Those of us at SAO are responsible for the design and development of the X-ray telescope for the Balloon-Borne Microcalorimeter Nuclear Line Explorer $(\boldsymbol{B}-\boldsymbol{M I N E})^{9,10}$. We are currently preparing a prototype multilayered telescope lens (Figure 10) for a test at the XACT facility in Palermo.

\section{CONCLUSIONS}

We have summarized the results of our plastic shell X-ray optics development program and presented them above. Our research program has progressed from small diameter spiral lenses intended for laboratory work to larger nested cylindrical lenses intended for astronomical applications. The results of an en evaluation of the experimental data obtained with these lenses have convinced us that the most promising approach for astronomical telescopes is a large, multilayered, single or double reflection spiral optic. This approach certainly offers a cost advantage and we shall know soon just how well it performs. Our first large lens is now being assembled and we will be tested soon. We shall report on the results at a future meeting.

\section{ACKNOWLEDGMENTS}

We thank Gerald K. Austin, David Caldwell, Richard Goddard, Lawrence J. Knowles and Warren Martell of SAO for their help with the engineering and technical aspects of the optics production. We also thank Giovanni Pareschi and Francesco Mazzoleni of Osservatorio Astronomico Di Brera for arranging the collaboration with SAO. IR\&D funding supported the research at SAO.

\section{REFERENCES}

1. H. W. Schnopper, M. Barbera, Eric Silver, R. Ingram, F. E. Christensen, S. Romaine, L. Cohen, A. Collura and S. S. Murray, Light weight, thin plastic foil, X-ray telescopes, Proc. SPIE 4496, 41 - 53, 2001.

2. H. W. Schnopper, M. Barbera, E. Silver, R. Ingram, F. E. Christensen, S. Romaine, L. Cohen, A. Collura, and S. S. Murray, Thin, Plastic shell, X-ray optics: an update, Proc SPIE 4851, 480 - 490, 2002.

3. H. W. Schnopper, M. Barbera, E. Silver, R. Ingram, F. E. Christensen, S. Romaine, L. Cohen, A. Collura and S. S. Murray, Light wight, thin plastic foil, X-ray telescopes, in Multi Color Universe Proceedings, 274 - 279, 2002, R. K. Manchanda and B. Paul, eds.

4. E. Silver, H. Schnopper, S. Bandler, S. Murray, N. Madden, D, Landis, J. Beeman, E. Haller, M. Barbera, G. Tucker, J. Gillaspy, E. Takacs and J. Porto, Laboratory astrophysics and microanalysis with NTD-germanium-based X-ray microcalorimeters, NIM A, 444, 156 - 160, 2000.

5. E. Silver, H. Schnopper, S. Bandler, N. Brickhouse, S. Murray, M. Barbera, E. Takacs, J. D. Gillaspy, J. V. Porto, I Kink, R Deslattes, L Hudson and J. M. Lamming, Laboratory Astrophysics Using a Microcalorimeter on an Electron Beam Trap, in X-Ray Astronomy 2000, ASP Conference Series, Vol. 234, 647 - 656, 2001, R. Giacconi, S. Serio and L. Stella eds.

6. H. W. Schnopper, S. Romaine and A. Krol, $X$-ray monochromator for divergent beam radiography using conventional and laser produced $X$-ray sources, Proc SPIE 4502, 19 - 28, 2001.

7. C. P. Jensen, private communication.

8. C. Liu, R. Conley, A. T. Marcrander, T. Graber, Ch. Morawe, C. Borel and E. M. Dufresne, Small-d-spacing WSi $\mathrm{C}_{2} / \mathrm{Si}$ narrow passband multilayers, Proc SPIE 5537, 2004 (in press).

9. H. Schnopper, E. Silver, S. Murray, C. Jones, W. Forman, S. Bandler, S. Romaine, P. Slane and J. Grindlay, B-MINE: Balloon-borne Microcalorimeter Nuclear Line Explorer, in X-Ray Astronomy 2000, ASP Conference Series, Vol. 234, 633 645, 2001, R. Giacconi, S. Serio and L. Stella eds.

10. E. Silver, H. Schnopper, C. Jones, W. Forman, S. Bandler, S. Murray, S. Romaine, P. Slane, J. Grindlay, N. Madden, J. Beeman, E. E. Haller, D. Smith, M. Barbera, A. Collura, F. Christensen, B. Ramsey, S. Woosley, R. Diehl, G. Tucker, J. Fabregat, V. Reglero and A. Gimenez, B-MINE, The Balloon-Borne Microcalorimeter Nuclear Line Explorer, Proc SPIE 4851, 905 - 912, 2002. 